\title{
Misturas asfálticas recicladas a quente com incorporação de elevado percentual de fresado como alternativa para camada de módulo elevado
}

\author{
Iran Gomes da Rocha Segundoํ, Verônica Teixeira Franco Castelo Branco², Kamilla L. Vasconcelos ${ }^{3}$ e Áurea \\ Silva de Holanda ${ }^{4}$
}

\begin{abstract}
Resumo: As Misturas Asfálticas de Módulo Elevado, do francês Enrobé à Module Élevé (EME), são constituídas por um Cimento Asfáltico de Petróleo (CAP) de baixa penetração e por agregados que compõem uma curva granulométrica densa, proporcionando as mesmas elevada resistência à deformação permanente e ao cisalhamento. As Misturas Asfálticas Recicladas a Quente (MARQ), com incorporação de elevado percentual de fresado, ou Reclaimed Asphalt Pavement (RAP), apresentam grande potencial de uso como material alternativo para a técnica mencionada (EME). Sua viabilidade é dada pelo fato de que o CAP oxidado, presente nessas misturas asfálticas, apresenta maior rigidez, além da vantagem relativa ao uso de uma matéria-prima de custo inferior a partir da reciclagem considerando elevados percentuais de uso da mesma. Este trabalho apresenta um estudo sobre o potencial de utilização de diferentes MARQ com incorporação de elevado percentual de fresado como camada de base e de ligação em pavimentos asfálticos, quais sejam: (i) Mistura I, composta exclusivamente por RAP como agregado; (ii) Mistura II, composta por 75\% de RAP e 25\% de agregados naturais; (iii) Mistura III, composta por 75\% de RAP e $25 \%$ de agregados do tipo refugo de usina de asfalto. Desta maneira, além da caracterização destes materiais em laboratório, foram realizadas análises computacionais, baseadas no Método dos Elementos Finitos (MEF). Os valores dos deslocamentos, das deformações e das tensões obtidos para diferentes estruturas de pavimentos asfálticos compostos por essas misturas foram avaliados a partir do dimensionamento de estruturas e, a partir disso, o tráfego que essas estruturas eram capazes de suportar foi calculado. Os resultados mostraram que as MARQ constituídas por elevado percentual de fresado podem compor estruturas utilizadas em rodovias de tráfego médio e alto.
\end{abstract}

Palavras-chave: reciclagem, alto percentual de fresado, refugo de usina de asfalto, misturas asfálticas de módulo elevado, dimensionamento de pavimentos.

\begin{abstract}
The high modulus asphalt mixtures (EME) are constituted by an asphalt binder of low penetration and aggregates that have a dense grading curve providing high resistance to permanent deformation and shear stresses. The recycled hot mixtures asphalt (RHMA) with the incorporation of high percentage of reclaimed asphalt pavement (RAP) have potential for the use as an alternative material for the mentioned technique (EME). Its viability is given by the fact that the RAP oxidized binder has higher stiffness (low penetration), and also by the economy that can be achieve with the use of raw material from recycling with potential lower cost. This paper presents a study of the potential use of different RHMA with high percentages of RAP as a base and intermediate course of pavements, such as: (i) Mixture I, composed exclusively by RAP as aggregate; (ii) Mixture II, composed by $75 \%$ of RAP and $25 \%$ of natural aggregates; (iii) Mixture III, composed by $75 \%$ of RAP and $25 \%$ of asphalt plant refuse aggregates. Thus, besides the characterization of these materials in the laboratory, computational analyzes were performed based on the Finite Element Method (FEM). The values of displacements, deformations and stresses obtained for the different asphalt pavement structures composed by these mixtures were evaluated and, from that, the traffic that they were able to withstand was calculated. The results showed that the high RAP RHMA could compose structures that can be used on medium and high volume traffic roads.
\end{abstract}

Keywords: recycling; High RAP Content; asphalt plant refuse aggregates; high modulus asphalt mixtures; pavement design.

\section{INTRODUÇÃO}

O fresado, do inglês Reclaimed Asphalt Pavement (RAP), é o principal resíduo gerado em serviços de pavimentação, sendo obtido através da fresagem de revestimentos asfálticos deteriorados. O refugo da usina de asfalto constitui outro resíduo importante dessa subárea da Engenharia Civil, sendo gerado em usinas de asfalto devido à

\footnotetext{
1 Iran Gomes da Rocha Segundo, Departamento de Engenharia de Transportes, UFC. (iran_gomes@hotmail.com)

2 Verônica Teixeira Franco Castelo Branco, Departamento de

Engenharia de Transportes, UFC. (veronica@det.ufc.br)

${ }^{3}$ Kamilla L. Vasconcelos, Departamento de Engenharia de Transporte,

USP. (kamilla.vasconcelos@gmail.com)

${ }^{4}$ Aurea Silva de Holanda, Departamento de Integração Acadêmica e

Tecnológica, UFC. (aurea@det.ufc.br)
}

Manuscrito recebido em 04/05/2016 e aprovado para publicação em 20/11/2016

Este artigo é parte de TRANSPORTES v. 24, n. 4, 2016. ISSN: 2237-1346 (online). DOI:10.14295/transportes.v24i4.1148 produção de misturas asfálticas fora das especificações de serviço, o que ocorre, geralmente, no início da produção das mesmas. Quando descartados de forma inadequada, geram um passivo ambiental e podem provocar uma série de danos à natureza. Ao incorporá-los em novas misturas asfálticas, pode-se economizar na estocagem dos mesmos e também no uso de materiais virgens (ligante asfáltico e agregados), diminuindo o dano ao meio ambiente.

As Misturas Asfálticas Recicladas a Quente (MARQ) com incorporação de alto percentual de RAP podem ser uma alternativa para uso em camadas de bases de pavimentos (Balbo e Bodi, 2004, Mallick et al., 2008, Ma et al., 2015). Há uma incerteza referente ao cálculo dos parâmetros volumétricos para essas misturas asfálticas. Para executar a técnica, existem algumas usinas capazes de produzilas e são utilizados os equipamentos convencionais para a sua aplicação (Zaumanis et al., 2014). 
ROCHA SEGUNDO, I.G.; CASTELO BRANCO, V.T.F.; VASCONCELOS, K.L.; HOLANDA, A.S.

\begin{tabular}{cc}
\hline Concreto asfáltico de desempenho elevado (ex: SMA) & $3,5 \mathrm{a} 7 \mathrm{~cm}$ \\
\hline Material de módulo elevado e resistente ao ATE & $10 \mathrm{a} 17 \mathrm{~cm}$ \\
\hline Material flexível e resistente à fadiga & 7 a $10 \mathrm{~cm}$ \\
\hline
\end{tabular}

(a) Pavimento de elevado desempenho e durabilidade (adaptado de Rohde, 2007)

\begin{tabular}{cc}
\hline BBTM & $2,5 \mathrm{~cm}$ \\
\hline BBME & $6 \mathrm{~cm}$ \\
\hline EME 2 & $9 \mathrm{~cm}$ \\
\hline EME 2 & $10 \mathrm{~cm}$ \\
\hline Subleito &
\end{tabular}

\begin{tabular}{cc}
\hline BBTM & $2,5 \mathrm{~cm}$ \\
\hline EME 2 & $12 \mathrm{~cm}$ \\
\hline & \\
Brita Simples & $20 \mathrm{~cm}$ \\
\end{tabular}

\section{(b) Pavimento sugerido por Corté e Guidoux (1998) \\ c) Pavimento sugerido por Scetauroute (1997) apud Corté (2001)}

Figura 1. Perfis estruturais constituídos por mistura de módulo elevado

Alguns países já possibilitam em sua legislação a utilização de até $100 \%$ de RAP em camadas de ligação ou de base, por exemplo, Alemanha, Áustria e Dinamarca segundo Direct-mat (2010) apud Cedex (2012).

Segundo Brosseaud (2001), é essencial para a fabricação de Misturas Asfálticas de Módulo Elevado, do francês, Enrobé à Module Élevé (EME), o uso de elevados percentuais de RAP. As EME, desenvolvidas na França desde a década de 80, são constituídas por Cimento Asfáltico de Petróleo (CAP) de baixa penetração e por agregados com curva granulométrica contínua, proporcionando uma elevada resistência à deformação permanente e ao cisalhamento. As EME são geralmente empregadas em camadas de ligação ou em bases para pavimentos especiais, mas não como revestimentos asfálticos por não atenderem aos critérios de superfície, principalmente relacionados com a textura das mesmas. Essas misturas asfálticas apresentam Módulo de Resiliência (MR) cerca de 2 a 3 vezes superiores se comparados aos valores encontrados para misturas asfálticas convencionais, são aplicadas com espessuras de 7 a $15 \mathrm{~cm}$ e são recomendadas para uso em vias urbanas com tráfego canalizado, rodovias de alto volume de tráfego e aeroportos (Motta et al., 2011). As EME podem apresentar MR entre 12.000MPa e 20.000MPa (Bernucci et al., 2007). Na norma espanhola PG-3 (OC 24/2008), as EME podem ser uma alternativa para uso em camadas de base ou de ligação. A utilização de RAP, porém, não deve ultrapassar $10 \%$ de incorporação. A espessura da camada deve ser maior que $7 \mathrm{~cm}$ e estas são destinadas a soluções para vias de médio e alto volumes de tráfego. O dimensionamento utilizando EME como camada de ligação ou de base pode reduzir em $25 \%$ a espessura da estrutura do pavimento (Engineer Journal, 2014), podendo reduzir em $24 \%$ as emissões em $\mathrm{kg} \mathrm{CO} \mathrm{CO}_{2} \mathrm{eq} / \mathrm{m}^{2}$ ano (Keijzer et al., 2015). Com isso, podese proporcionar economia na utilização de recursos naturais, no transporte de material da usina à obra e no tempo de execução de camadas.

O pavimento perpétuo, do inglês full depth asphalt, consiste em um pavimento composto por camadas de misturas asfálticas: revestimento resistente ao cisalhamento, base composta por mistura asfáltica densa com elevado módulo de elasticidade e subbase composta por mistura asfáltica flexível, resistente ao trincamento por fadiga (Balbo e Bodi, 2004) e com valores baixos de deformações por tração no fundo da camada de rolamento. Essa tecnologia é normalmente utilizada para um pavimento com vida útil de 50 anos, necessitando de manutenções com profundidade de 2,5 a $10 \mathrm{~cm}$, aproximadamente, a cada 20 anos (Villacorta, 2005). Nos pavimentos flexíveis tradicionais, as trincas por fadiga ocorrem, normalmente, motivadas pela geração de tensão de tração na fibra inferior da camada de revestimento. Por outro lado, com a execução de pavimentos perpétuos, a propagação dessa patologia pode ser minimizada, uma vez que as trincas não conseguem alcançar elevadas profundidades (Maupin e Diefenderfer, 2006).

Rohde (2007) apresenta um perfil estrutural para pavimentos de elevado desempenho e durabilidade sendo como estrutura: (i) concreto asfáltico de desempenho elevado, por exemplo, Stone Matrix Asphalt (SMA); (ii) material de módulo elevado resistente à deformação permanente, ou seja, ao Afundamento de Trilha de Roda (ATR); (iii) material flexível e resistente à fadiga (Figura 1a). Segundo Corté e Guidoux (1998), uma alternativa para o dimensionamento utilizando EME para vias de tráfego pesado (20 milhões de eixos equivalentes a $130 \mathrm{kN}$ ) sobre um subleito com módulo de 120MPa teria o seguinte perfil: (i) camada 
de rolamento de 2,5cm de BBTM (Béton Bitumineux Très Mince - mistura asfáltica delgada de granulometria descontínua); (ii) camada de ligação de $6 \mathrm{~cm}$ de BBME (Betón Bitumineux à Module Élevé - mistura asfáltica de módulo elevado para revestimento); (iii) base de $9 \mathrm{~cm}$ de EME 2 (Classe 2 da especificação francesa, que caracteriza uma mistura asfáltica com elevada resistência ao trincamento por fadiga); (iv) subbase de $10 \mathrm{~cm}$ composta por EME 2 (Figura $1 b)$.

Segundo o Catálogo Scetauroute (1997) apud Corté (2001), uma alternativa para o dimensionamento de um pavimento submetido a 600 veículos pesados/dia, $4 \%$ de taxa de crescimento anual, período de projeto de 15 anos e subleito com módulo de $120 \mathrm{MPa}$ seria: (i) camada de rolamento de 2,5cm de BBTM; (ii) base de $12 \mathrm{~cm}$ de EME 2; (iii) subbase de $20 \mathrm{~cm}$ de brita simples (Figura 1c).

Magalhães (2004) realizou análises estruturais em pavimentos compostos por EME como camada de ligação a partir do uso de diferentes ligantes asfálticos para um tráfego de $10^{8}$ (repetições do eixo padrão). A solução para o dimensionamento da estrutura trata-se da seguinte: (i) revestimento composto por SMA com $4 \mathrm{~cm}$ de espessura; (ii) camada de ligação composta por EME com $12 \mathrm{~cm}$ de espessura (utilizando CAP modificado por EVA) ou de $8 \mathrm{~cm}$ (utilizando resíduo asfáltico de petróleo proveniente do petróleo árabe por desasfaltação a propano como ligante asfáltico - RASF); (iii) camada de base composta por Brita Graduada Tratada com Cimento (BGTC) com $15 \mathrm{~cm}$ de espessura, gerando um pavimento $24 \%$ e $34 \%$, respectivamente, mais delgado se comparado ao pavimento ao pavimento utilizado na rodovia Carvalho Pinto (SP-70), que utiliza revestimento constituído por Concreto Asfáltico (CA), base constituída por Brita Graduada Simples (BGS) e subbase constituída por BGTC.

Freitas e Motta (2008) avaliaram o potencial de uso da escória de aciaria em diferentes misturas asfálticas do tipo EME através de ensaios mecânicos e análises estruturais. Com relação à análise estrutural para um tráfego de $10^{8}$, os autores propuseram o seguinte dimensionamento: (i) revestimento composto por SMA com $3 \mathrm{~cm}$; (ii) camada de ligação composta por EME de espessura de 6 a $8 \mathrm{~cm}$ (a depender do CAP utilizado); (iii) base composta por BGS de $12 \mathrm{~cm}$, resultando em um pavimento $44 \%$ e $49 \%$, respectivamente, mais delgado se comparado ao pavimento utilizado na Rodovia Carvalho Pinto.

O método empírico de dimensionamento com o uso do Índice de Suporte Califórnia (do inglês, California Bearing Ratio - CBR) é utilizado no Brasil desde a década de 60. A espessura das camadas dos pavimentos flexíveis é definida por meio de equações empíricas a partir do conhecimento do valor do CBR do subleito. Para que seja feito um dimensionamento mais realista dos pavimentos, é importante a realização de uma análise estrutural, com o uso de modelos de previsão de desempenho, o que já vem sendo adotado em diversos países (como o Mechanistic Empirical Pavement Design Guide dos EU - MEPDG) e mesmo por alguns departamentos de transportes no Brasil, como é o caso do DER-SP.

No MEPDG, são inseridos em um software dados: relativos ao carregamento de acordo com o tráfego, dos materiais (módulo de elasticidade, coeficiente de Poisson e ângulo de fase, este para o caso de uma análise viscoelástica) e as espessuras das camadas para o cálculo, através do uso de modelos analíticos, das tensões, das deformações e dos deslocamentos em qualquer ponto da estrutura. Os resultados de zonas críticas, topo do revestimento, fibra inferior do revestimento e topo do subleito, são comparados com os valores permitidos de tensões, de deformações e de deslocamentos. Caso estes não sejam satisfeitos, são realizadas novas tentativas até que os requisitos sejam atendidos (NCHPR 1-37, 2004; Muthadi, 2008; Aragão et al., 2010; Selvi, 2015).

No modelo de previsão francês, também utilizado para misturas asfálticas de módulo elevado, o número de ciclos admissíveis (NE) leva em consideração a deformação horizontal admissível no fundo da camada asfáltica $\left(\varepsilon_{\mathrm{t}, \mathrm{adm}}\right)$; o coeficiente de risco $\left(\mathrm{k}_{\mathrm{r}}\right)$, escolhido pelo valor de tensão ao considerar um intervalo de confiança sobre as espessuras e os valores de fadiga; o coeficiente de ajuste $\left(\mathrm{k}_{\mathrm{c}}\right)$, o qual leva em consideração o desempenho do pavimento real; o coeficiente de redução $\left(\mathrm{k}_{\mathrm{s}}\right)$, que leva em consideração a falta de uniformidade do subleito; a deformação à fadiga do material $\left(\varepsilon_{6}\right)$ após $10^{6}$ ciclos de carregamento, temperatura de $10^{\circ} \mathrm{C}$ e $25 \mathrm{~Hz}$; os módulos de rigidez a $10^{\circ} \mathrm{C}\left(\mathrm{E}\left(10^{\circ} \mathrm{C}\right)\right)$ e a temperatura de projeto $(\mathrm{E}(\theta))$ (Equação 1) (Perret et al., 2004; Balay et al., 2012; Njock e Yueguang, 2015).

$$
N E=\left[\frac{\varepsilon_{6}\left(10^{\circ} \mathrm{C}, 25 \mathrm{~Hz}\right) \times k_{r} \times k_{c} \times k_{s}}{\varepsilon_{t, a d m}} \times\left(\frac{E\left(10^{\circ} \mathrm{C}\right)}{E(\theta)}\right)^{0,5}\right]^{\frac{-1}{0,2}}
$$

Timm e Newcomb (2016) e Newcomb et al. (2010) se preocuparam com a caracterização e com o dimensionamento de pavimentos especiais e desenvolveram um programa de dimensionamento para pavimentos perpétuos. $\mathrm{O}$ modelo faz o uso das Equações (2) e (3) para fadiga e deformação permanente, respectivamente. Nf é o tráfego, $\varepsilon t$ a deformação horizontal de tração, E o módulo de elasticidade da mistura asfáltica e $\varepsilon v$ a deformação vertical de compressão.

$$
\begin{gathered}
\log N f=15,947-3,291 \log \left(\frac{\varepsilon t}{10^{-6}}\right)-0,854 \log \left(\frac{E}{10^{3}}\right) \\
N f=1,077 \times 10^{18}\left(\frac{10^{-6}}{\varepsilon v}\right)
\end{gathered}
$$

Neste artigo, foram realizadas análises computacionais baseadas no Método dos Elementos Finitos (MEF) para avaliar o potencial de uso de três misturas asfálticas do tipo MARQ com incorporação de elevado percentual de RAP como camada de ligação e como camada de base: (i) Mistura I, composta exclusivamente por RAP como agregado; (ii) Mistura II, composta por $75 \%$ de RAP e $25 \%$ de agregados naturais; (iii) Mistura III, composta por $75 \%$ de RAP e $25 \%$ de refugo de usina de asfalto. A partir dos valores de MR de cada uma das misturas asfálticas, foi realizado um estudo paramétrico para a determinação dos valores dos deslocamentos, das deformações e das tensões obtidos e, em seguida, foi calculado o tráfego (número N) que as estruturas estudadas eram capazes de suportar. 
ROCHA SEGUNDO, I.G.; CASTELO BRANCO, V.T.F.; VASCONCELOS, K.L.; HOLANDA, A.S.

Tabela 1. Resultados das dosagens das MARQ com incorporação de elevado percentual de RAP

\begin{tabular}{ccccccccccc}
\hline $\begin{array}{c}\text { Mistura } \\
\text { asfáltica }\end{array}$ & \% RAP & \% refugo & $\begin{array}{c}\text { \% brita } \\
\mathbf{3 / 8}\end{array}$ & $\begin{array}{c}\text { Teor de CAP } \\
\text { total (\%) }\end{array}$ & $\begin{array}{c}\text { Teor de CAP } \\
\text { virgem (\%) }\end{array}$ & Gmm & Gmb & Vv (\%) & $\begin{array}{c}\text { RBV } \\
(\boldsymbol{\%})\end{array}$ \\
\hline I & 100 & - & - & 5,2 & 0,3 & 2,474 & 2,370 & 4,3 & 68,3 \\
II & 75 & - & 25 & 4,9 & 1,3 & 2,407 & 2,309 & 4,1 & 71,4 \\
III & 75 & 25 & - & 5,3 & 1,1 & 2,461 & 2,363 & 4,0 & 71,7 \\
\hline
\end{tabular}

Tabela 2. Resultados dos ensaios de Módulo de Resiliência (MR) e de Resistência à Tração (RT)

\begin{tabular}{|c|c|c|c|c|c|c|c|}
\hline \multirow[b]{2}{*}{ Parâmetro } & \multicolumn{3}{|c|}{ Mistura estudadas nesta pesquisa } & \multirow{2}{*}{$\begin{array}{l}\text { Mistura Referência } \\
\text { de Oliveira (2013) }\end{array}$} & \multirow{2}{*}{\multicolumn{3}{|c|}{$\begin{array}{l}\text { MARQ avaliada por Oliveira } \\
\text { (2013) }\end{array}$}} \\
\hline & Mistura I & Mistura II & Mistura III & & & & \\
\hline$\%$ RAP & 100 & 75 & 75 & - & 15 & 35 & 50 \\
\hline$\%$ CAP virgem & 0,3 & 1,3 & 1,1 & 5,0 & 4,0 & 3,0 & 2,3 \\
\hline$\%$ CAP total & 5,2 & 4,9 & 5,3 & 5,0 & 4,8 & 5,5 & 5,2 \\
\hline $\mathrm{Vv}(\%)$ & 4,3 & 4,0 & 3,9 & 5 & 3,7 & 3,9 & 3,8 \\
\hline $\mathrm{MR}(\mathrm{MPa})$ & 16.003 & 14.487 & 14.812 & 3.809 & 6.056 & 7.144 & 8.435 \\
\hline $\mathrm{RT}(\mathrm{MPa})$ & 3,1 & 2,9 & 2,9 & 0,9 & 1,4 & 1,8 & 2,2 \\
\hline Desgaste Cântabro (\%) & 11 & 10 & 10 & $*$ & $*$ & $*$ & $*$ \\
\hline
\end{tabular}

${ }^{*}$ Ensaio não realizado no trabalho

\section{MATERIAIS}

\subsection{Cimento Asfáltico de Petróleo}

Foi utilizado nesta pesquisa, como ligante, o CAP fornecido pela Petrobras/Lubnor proveniente do campo Fazenda Alegre. Este ligante foi caracterizado pelo ensaio de penetração como CAP 50/70 (DNIT-ME 155/2010) e com grau de desempenho PG 70-28 segundo ASTM D6373 (2013).

Ademais, esse CAP apresenta as seguintes características: i) viscosidade Brookfield a $60^{\circ} \mathrm{C}$ de $2.415 \mathrm{P}$; ii) Ponto de Amolecimento de $48^{\circ} \mathrm{C}$; iii) Penetração Retida de $75 \%$ e aumento do Ponto de Amolecimento de $3,8^{\circ} \mathrm{C}$ após Rolling Thin Film Ovens (RTFOT).

\subsection{Agregados}

$\mathrm{O}$ agregado virgem utilizado nesta pesquisa foi a brita de $3 / 8$ " de origem fonolítica, fornecida pela pedreira OCS, que se localiza na Região Metropolitana de Fortaleza (RMF). O RAP utilizado como agregado nesta pesquisa tem origem no processo de fresagem padrão de vias da RMF. Vale ressaltar a sua extrema heterogeneidade, sendo necessária a caracterização mais frequente em escala real com a finalidade de reduzir a variabilidade do mesmo de forma a obter-se dosagens mais representativas.

Esse material apresentou 5,0\% de CAP (DNER-ME 053/94), o qual não foi caracterizado por não ser o foco deste estudo. O refugo da usina de asfalto utilizado na Mistura III trata-se de toda a massa asfáltica produzida fora das especificações de serviço em termos de: granulometria, teor de ligante asfáltico, envolvimento do material e/ou temperatura. $\mathrm{O}$ refugo apresentou $2,1 \%$ de CAP (DNER-ME 053/94).

Os resíduos foram cedidos pela empresa Insttale Engenharia Ltda., a mesma que cedeu o RAP utilizado na pesquisa desenvolvida por Oliveira (2013), cujos resultados serão utilizados a título de comparação no presente estudo.

\subsection{Misturas Asfálticas}

Foram avaliadas MARQ (enquadradas na faixa $\mathrm{C}$ para CA do DNIT), todas com Tamanho Máximo Nominal (TMN) de 12,5mm: (i) Mistura I - mistura asfáltica composta por $100 \%$ de RAP; (ii) Mistura II - mistura asfáltica composta por $75 \%$ de RAP e $25 \%$ de brita de $3 / 8$ "; (iii) Mistura III: mistura asfáltica composta por $75 \%$ de RAP e $25 \%$ de refugo de usina.

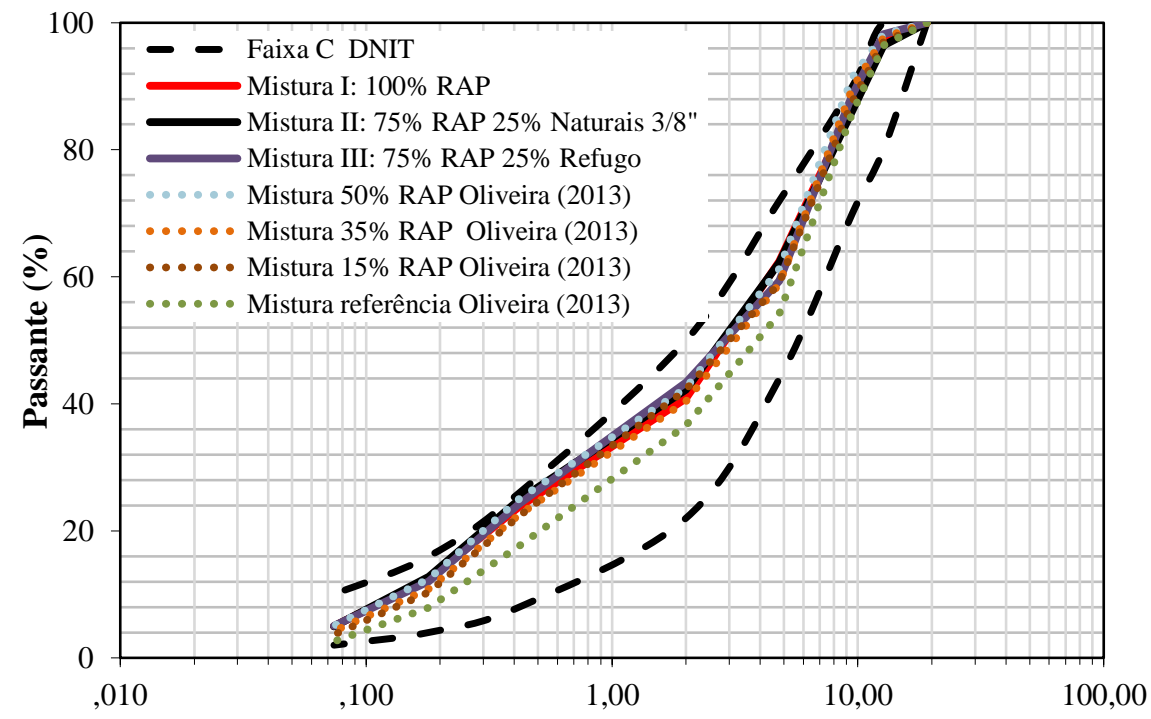

Abertura das Peneiras (mm)

Figura 2. Curvas granulométricas das misturas asfálticas avaliadas neste trabalho e por Oliveira (2013) 


\begin{tabular}{|cccc|}
\hline Capa - SMA & MR $=2.200 \mathrm{MPa}$ & $\mu=0,30$ & $\mathrm{~h}=4 \mathrm{~cm}$ \\
\hline $\begin{array}{c}\text { Ligação }- \text { MARQ elevado } \\
\text { percentual de RAP }\end{array}$ & MR = variável & $\mu=0,30$ & $\mathrm{~h}=8 \mathrm{~cm}$ \\
\hline Base - BGTC & MR $=10.000 \mathrm{MPa}$ & $\mu=0,20$ & $\mathrm{~h}=15 \mathrm{~cm}$ \\
& & & \\
\hline Subleito & $\mathrm{MR}=150 \mathrm{MPa}$ & $\mu=0,45$ & $\mathrm{~h}=$ infinito \\
\hline
\end{tabular}

(a) Perfil (3a) - Alternativa para uso de MARQ em camada de ligação

\begin{tabular}{|cccc|}
\hline Capa - SMA & MR $=2.200 \mathrm{MPa}$ & $\mu=0,30$ & $\mathrm{~h}=4 \mathrm{~cm}$ \\
$\begin{array}{c}\text { Ligação }- \text { Concreto } \\
\text { Asfáltico Faixa B }\end{array}$ & MR $=3.000 \mathrm{MPa}$ & $\mu=0,30$ & $\mathrm{~h}=8 \mathrm{~cm}$ \\
\hline $\begin{array}{c}\text { Base - MARQ elevado } \\
\text { percentual de RAP }\end{array}$ & $\mathrm{MR}=$ variável & $\mu=0,30$ & $\mathrm{~h}=15 \mathrm{~cm}$ \\
\hline Subleito & $\mathrm{MR}=150 \mathrm{MPa}$ & $\mu=0,45$ & $\mathrm{~h}=$ infinito \\
\hline
\end{tabular}

(b) Perfil (3b) - Alternativa para uso de MARQ em camada de base Figura 3: Perfis avaliados

Todas as misturas asfálticas possuem a mesma granulometria da MARQ confeccionada por $50 \%$ de fresado e semelhantes às demais MARQ avaliadas por Oliveira (2013) (Figura 1). O autor avaliou MARQ compostas por $15 \%, 35 \%$ e $50 \%$ de RAP, além de uma mistura asfáltica de referência, sem a presença de RAP.

As misturas asfálticas avaliadas neste trabalho foram dosadas pelo método Superpave buscando um Volume de vazios (Vv) de $4 \%$ para amostras com $10 \mathrm{~cm}$ de diâmetro e $6,35 \mathrm{~cm}$ de altura compactadas com 100 giros. As amostras foram dosadas com a temperatura de usinagem de $160^{\circ} \mathrm{C}$ para o CAP e de $175^{\circ} \mathrm{C}$ para os agregados. A temperatura de compactação das misturas asfálticas foi de $155^{\circ} \mathrm{C}$.

Essas temperaturas são as mesmas temperaturas utilizadas por Oliveira (2013), obtidas através da curva de viscosidade versus temperatura para o CAP utilizado nesse estudo. Sabe-se, porém, que, devido ao fato do CAP presente no RAP ser mais viscoso do que o CAP virgem, aquele requer Temperaturas de Usinagem e de Compactação (TUC) mais elevadas, o que não foi considerado nesse estudo devido à dificuldade de recuperação do CAP contido no resíduo. Ressalta-se, porém, que a mesma simplificação foi feita para todos os materiais estudados na presente pesquisa e por Oliveira (2013) o que, de certa forma, mantém a possibilidade de comparação dos resultados encontrados.

São apresentados na Tabela 1 a percentagem em massa dos agregados e dos teores de CAP total e virgem assim como os seguintes parâmetros volumétricos obtidos para as misturas asfálticas compactadas no teor de projeto de CAP: massa específica máxima medida $\left(\mathrm{G}_{\mathrm{mm}}\right)$, massa específica aparente da mistura asfáltica compactada $\left(\mathrm{G}_{\mathrm{mb}}\right)$, Volume de vazios (Vv) e Relação Betume/Vazios (RBV). As misturas asfálticas foram avaliadas quanto à Resistência à Tração Indireta (RT) (DNIT-ME 136), ao Módulo de Resiliência (MR) (NBR 16018) e ao Desgaste Cântabro (DNER 386) (Tabela 2).

As MARQ apresentaram praticamente o mesmo teor de CAP total, o mesmo Vv e a mesma RBV. A Mistura I apresenta provavelmente um maior percentual de CAP envelhecido, enquanto a Mistura III apresenta a combinação de três CAP: CAP virgem e CAP presentes no fresado e no refugo da usina.

As Misturas I, II e III apresentaram valores de RT 3,22 a 3,44 vezes superiores e de MR 3,89 a 4,20 vezes superiores se comparados ao valor obtido para a mistura de referência avaliada por Oliveira (2013). O percentual de fresado apresentou uma tendência linear com o RT de $R^{2}$ de 0,99 da mesma maneira com o MR de $R^{2}$ de 0,95 .

\section{METODOLOGIA}

Foram realizadas análises de estruturas com utilização do software PMG/CAP3D desenvolvido no Laboratório de Mecânica dos Pavimentos (LMP)/UFC. A geração da malha de elementos finitos foi realizada pelo pré-processador PMG (Pavement Mesh Generator) (Cavalcante et al., 2008; Coutinho et al., 2008; Botelho et al., 2009), enquanto a análise linear elástica foi realizada pelo CAP3D (Computational Analysis of Pavements) (Holanda et al., 2006).

Foram utilizados elementos quadráticos de 8 nós (Q8) para os elementos finitos e elementos bidimensionais Lagrangeanos de 6 nós (L6) para os elementos infinitos para a geração de uma malha axissimétrica mista conforme apresentado por Silva et al. (2013). 
ROCHA SEGUNDO, I.G.; CASTELO BRANCO, V.T.F.; VASCONCELOS, K.L.; HOLANDA, A.S.

Tabela 3. Equações e parâmetros utilizados para o cálculo do número $\mathrm{N}$

\begin{tabular}{|c|c|c|c|c|c|}
\hline Local de Análise & Equação & $\mathrm{K}$ & $n$ & $\sigma r(\mathrm{MPa})$ & Fonte \\
\hline $\begin{array}{l}\text { Fibra Inferior do } \\
\text { Revestimento (4) }\end{array}$ & $N_{i r}=\mathrm{k} \times-$ & $1,092 \times 10^{-6}$ & 3,512 & - & $\begin{array}{c}\text { FHWA (1976) apud } \\
\text { DER-SP (2006) }\end{array}$ \\
\hline
\end{tabular}

\begin{tabular}{|c|c|c|c|c|}
\hline $\begin{array}{l}\text { Topo do Subleito } \\
\text { (5) }\end{array}$ & $N_{s u b}=\mathrm{k} \times$ & $6,069 \times 10^{-10}$ & 4,762 & $\begin{array}{l}\text { Dormon e Metcalf } \\
\text { (1965) apud DER-SP } \\
\text { (2006) }\end{array}$ \\
\hline
\end{tabular}

Fibra Inferior da

Base Tratada com Cimento (6)

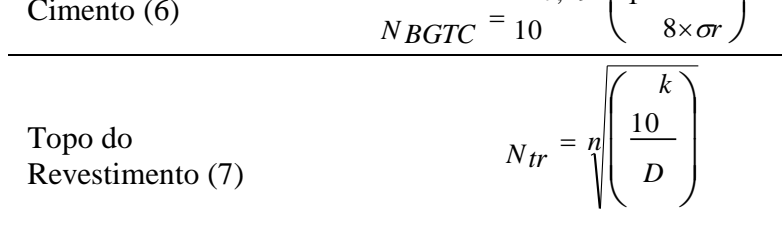

Foram analisadas duas estruturas (Figura 3) com a presença das MARQ com incorporação de elevado percentual de RAP como alternativa para uma camada de: (a) ligação (Perfil (3a) apresentado na Figura 3a); (b) base (Perfil (3b) apresentado na Figura 3b). Vale informar que as camadas cimentadas geralmente são analisadas em duas fases: íntegra, considerada neste trabalho, e após trincamento.

Nas análises, foi considerada a aderência perfeita entre as camadas (sem deslizamento). Foi aplicada uma pressão de pneu de $0,56 \mathrm{MPa}$ em um raio de atuação de $10,8 \mathrm{~cm}$ (representando a carga de uma roda do eixo padrão de $80 \mathrm{kN}$ ) para obter os valores de: (i) deformação horizontal de tração na fibra inferior da camada de revestimento $\left(\varepsilon t_{r}\right)$ e da camada de ligação $\left(\varepsilon t_{l}\right)$; (ii) deformação vertical no topo do subleito $(\varepsilon v)$; (iii) tensão de tração na fibra inferior da base do tipo BGTC ( $\sigma t$, apenas para o Perfil (3a)); (iv) deslocamento vertical no topo do revestimento (D). Salienta-se que todos os resultados obtidos foram analisados diretamente abaixo do carregamento.

Após realizada a análise estrutural, o número equivalente de operações de eixo simples de rodas duplas de $80 \mathrm{kN}$ acumulado para um período de projeto $(\mathrm{N})$, o qual o perfil é capaz de suportar, será calculado. Serão utilizadas as Equações de (4) a (7) (DER-SP, 2006) e os valores de k, $n$ e $\sigma r$ segundo a Tabela 3. Vale salientar que, das equações apresentadas na Tabela 3, a Equação (4) faz referência aos fatores de equivalência de carga (FEC) da AASHTO para a determinação do tráfego, ou $\mathrm{N}_{\text {previsto, }}$ e as Equações (5), (6) e (7) fazem referência aos FEC da USACE. Os fatores de equivalência de carga da USACE e da AASHTO encontram-se descritos no "Manual de Restauração de Pavimentos Asfálticos" do DNIT (2006).

Conforme reportado por Pinto e Preusller (2010), os FEC da AASHTO baseiam-se na perda de serventia e variam com o tipo do pavimento (flexível e rígido), índice de serventia terminal e resistência do pavimento (número estrutural - SN).

O FEC da AASHTO difere do da USACE, onde avaliaram-se os efeitos do carregamento na resistência a deformação permanente.
Em que:

$\mathrm{N}_{\text {ir: }}$ : N para o a fibra inferior do revestimento, sendo subclassificado como $\mathrm{N}_{\text {irr }}$ e $\mathrm{N}_{\text {irl }}$ para respectivamente à camada de rolamento e à camada de ligação;

$\mathrm{N}_{\text {sub }}$ : N para o topo do subleito;

$\mathrm{N}_{\text {BGTC: }} \mathrm{N}$ para a Base Tratada com Cimento (BTC); $\mathrm{N}_{\mathrm{tr}}$ : N para o topo do revestimento;

$\varepsilon t$ : deformação específica horizontal de tração, sendo subclassificado como $\varepsilon t_{\mathrm{r}}$ e $\varepsilon t_{1}$ para respectivamente à camada de rolamento e à camada de ligação;

$\varepsilon v$ : deformação específica vertical de compressão;

$\sigma t$ : tensão de tração atuante (MPa);

$\sigma r$ : tensão de tração na ruptura do material (MPa);

D: deslocamento vertical/deflexão (mm);

k e $n$ : coeficientes determinados por regressões lineares, particulares para cada tipo de mistura asfáltica e modificados para refletir o desempenho no campo.

\section{RESULTADOS E DISCUSSÕES}

São apresentados na Tabela 4 a análise estrutural e os valores de $\mathrm{N}$ que o Perfil (3a) é capaz de suportar para a carga solicitada.

Nota-se que os valores de $\mathrm{N}$ permaneceram praticamente os mesmos alterando apenas o MR da camada de ligação para as Misturas II e III, exceto para a análise realizada na fibra inferior da camada de base, que foram iguais. Quanto aos resultados de $\mathrm{N}$ para as três misturas asfálticas com o uso de RAP, o tráfego máximo que é de $1,07 \times 10^{7}$, tendo como ponto crítico a camada de base constituída por BGTC ( $\left.\mathrm{N}_{\text {BGTC }}\right)$. O N, calculado pelos demais critérios $\left(\mathrm{N}_{\mathrm{irl}}\right.$, $\mathrm{N}_{\text {sub }}$ e $\mathrm{N}_{\text {tr }}$ ), apresentou ordem de grandeza pelo menos 1.000 vezes superior. Ademais, o $\mathrm{N}_{\text {irr }}$, calculado pela $\varepsilon t_{r}$, referente à deformação de tração horizontal na fibra inferior da camada de rolamento, não foi calculado devido à deformação de compressão apresentada no cálculo ter apresentado valor negativo.

O Perfil (3a) apresenta estrutura constituída pelos mesmos materiais do pavimento avaliado por Magalhães (2004) e igual espessura das camadas quando a solução apresenta camada de ligação constituída por EME com RASF como ligante asfáltico. 
Misturas Asfálticas Recicladas a Quente com Incorporação de Elevado Percentual de Fresado Como Alternativa para Camada de Módulo Elevado

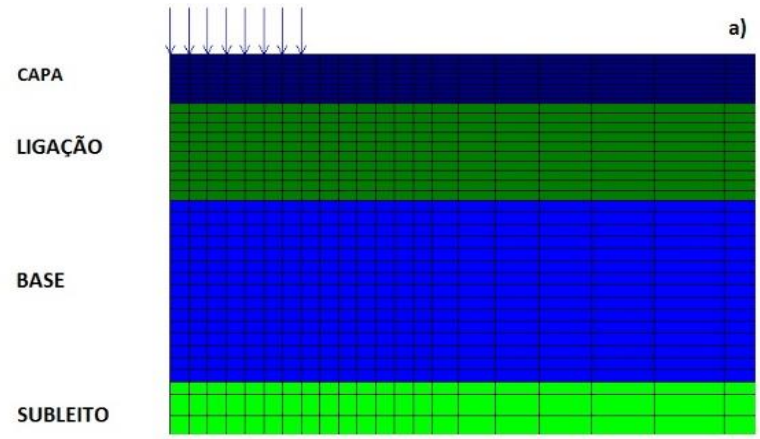

(a) Malha e camadas

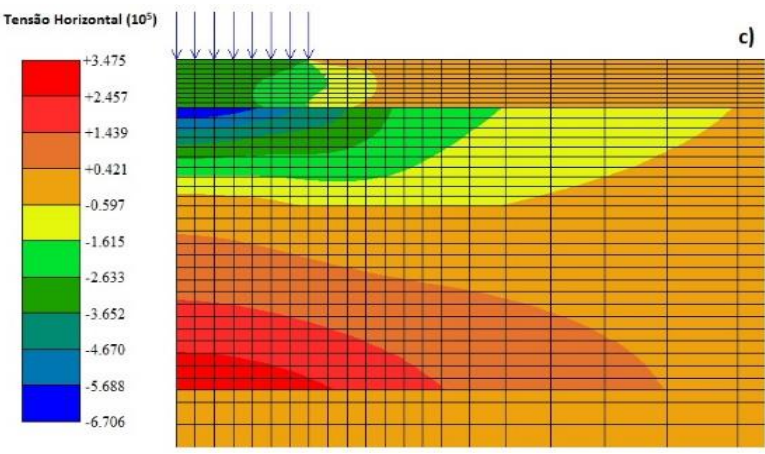

(c) Tensão horizontal $(\mathrm{Pa})$

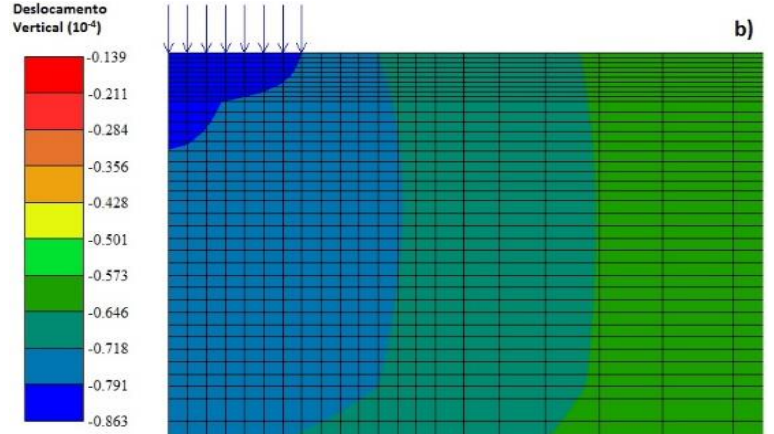

(b) Deslocamento vertical (m)

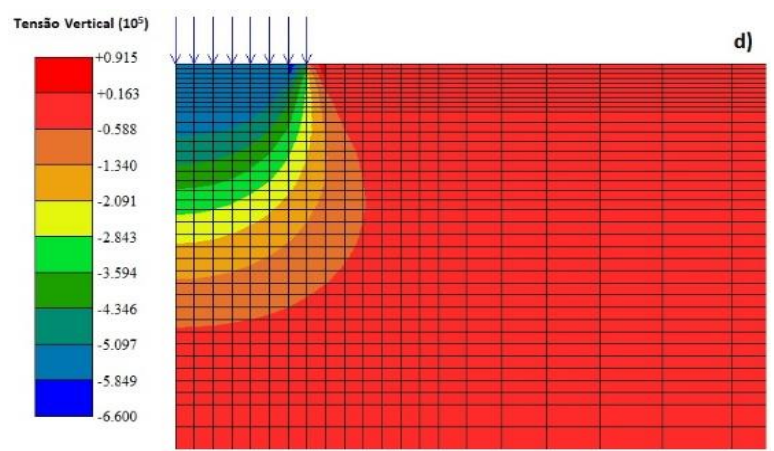

(d) Tensão vertical $(\mathrm{Pa})$

Figura 4. Detalhe da região próxima à carga para o Perfil (3a) que utiliza a Mistura I como camada de ligação

No entanto, o tráfego que a estrutura é capaz de suportar tem ordem de grandeza 10 vezes inferior. Como os resultados para as diferentes misturas foram semelhantes, para melhor visualizar os resultados, na Figura 4 é apresentada uma representação gráfica dos resultados obtidos para o deslocamento vertical e para as tensões horizontal e vertical do Perfil (3a) cuja Mistura I compõe a camada de ligação.

Tabela 4. Resultados da análise estrutural para o Perfil (3a): alternativa para uso da MARQ em camada de ligação

\begin{tabular}{|c|c|c|c|}
\hline Mistura & Mistura I & Mistura II & Mistura III \\
\hline$\overline{\varepsilon t_{r}}$ & $-1,91 \times 10^{-5 *}$ & $-1,96 \times 10^{-5 *}$ & $-1,95 \times 10^{-5 *}$ \\
\hline$\varepsilon t_{l}$ & $5,37 \times 10^{-6}$ & $4,83 \times 10^{-6}$ & $4,96 \times 10^{-6}$ \\
\hline$\varepsilon v$ & $6,88 \times 10^{-5}$ & $6,99 \times 10^{-5}$ & $6,97 \times 10^{-5}$ \\
\hline$\sigma t(\mathrm{MPa})$ & $\mathbf{0 , 3 5}$ & $\mathbf{0 , 3 5}$ & $\mathbf{0 , 3 5}$ \\
\hline $\begin{array}{l}\mathrm{D}\left(10^{-2}\right. \\
\mathrm{mm})\end{array}$ & 8,63 & 8,73 & 8,71 \\
\hline $\mathrm{N}_{\mathrm{irr}}{ }^{* * * *}$ & $* *$ & $* *$ & $* *$ \\
\hline $\mathrm{N}_{\mathrm{irl}}{ }^{* * * *}$ & $3,52 \times 10^{12}$ & $5,10 \times 10^{12}$ & $4,67 \times 10^{12}$ \\
\hline $\mathrm{N}_{\text {sub }}$ & $4,02 \times 10^{10}$ & $3,73 \times 10^{10}$ & $3,79 \times 10^{10}$ \\
\hline $\mathrm{N}_{\mathrm{BGCT}}$ & $1,07 \times 10^{7}$ & $1,07 \times 10^{7}$ & $1,07 \times 10^{7}$ \\
\hline $\mathrm{N}_{\mathrm{tr}}$ & $8,30 \times 10^{11}$ & $7,78 \times 10^{11}$ & $7,86 \times 10^{11}$ \\
\hline
\end{tabular}

*O valor negativo representa deformação de compressão.

** A equação utilizada fornece o valor de $\mathrm{N}$ para uma deformação de tração.

*** Uma vez que o N calculado a partir da deformação de tração na fibra inferior do revestimento é levado em consideração no cálculo do FEC da AASHTO, este não pode ser diretamente comparado aos demais valores de $\mathrm{N}\left(\mathrm{N}_{\mathrm{sub}}, \mathrm{N}_{\mathrm{BGTC}}\right.$ e $\left.\mathrm{N}_{\mathrm{tr}}\right)$.

Pela representação gráfica apresentada na Figura 4, é possível verificar que a concentração de tensão horizontal de tração está localizada na fibra inferior da base cimentada, local onde há a ruptura do pavimento. A tensão vertical é dissipada, iniciando seu valor mínimo e constante na metade inferior da base. Ao comparar os resultados do perfil com o valor de N, calculado a partir de FEC da USACE, das rodovias estaduais CE-060 - Pajuçara - Pacatuba $(\mathrm{N}=$ $\left.3,74 \times 10^{6}\right)$ e CE-040 - Messejana - Aquiraz $\left(\mathrm{N}=7,43 \times 10^{6}\right)$, acredita-se que o Perfil (3a) é capaz de resistir ao tráfego de rodovias com volume de tráfego médio.

As mesmas rodovias apresentariam, considerando o valor do FEC da AASHTO, valores de $\mathrm{N}$ em torno de $3,63 \times 10^{6}$ e $6,41 \times 10^{6}$, respectivamente. Ademais, para rodovias nacionais brasileiras, o valor de $\mathrm{N}$ do Perfil (3a) tem a mesma ordem de grandeza daquele encontrado para o caso da BR-116 - Fortaleza - Pacajus, que é de $\mathrm{N}=3,84 \times 10^{7}$, obtido a partir de valores de FEC da USACE, e aproximadamente de $2,66 \times 10^{7}$, obtido a partir de valores de FEC da AASHTO (Benevides, 2000; Benevides, 2005).

São apresentados na Tabela 5 a análise estrutural e os valores de $\mathrm{N}$ que o Perfil (3b) é capaz de suportar para a carga solicitada.

Tabela 5. Resultados da Análise Estrutural para o Perfil (3b): alternativa para uso da MARQ em uma camada de Base

\begin{tabular}{|c|c|c|c|}
\hline Mistura & Mistura I & Mistura II & Mistura III \\
\hline$\varepsilon t_{r}$ & $-8,56 \times 10^{-6 *}$ & $-9,70 \times 10^{-6 *}$ & $-9,45 \times 10^{-6 *}$ \\
\hline$\varepsilon t_{l}$ & $-7,68 \times 10^{-6 *}$ & $-7,41 \times 10^{-6 *}$ & $-7,48 \times 10^{-6 *}$ \\
\hline$\varepsilon v$ & $6,95 \times 10^{-5}$ & $7,31 \times 10^{-5}$ & $7,23 \times 10^{-5}$ \\
\hline $\mathrm{D}\left(10^{-2} \mathrm{~mm}\right)$ & 10,2 & 10,4 & 10,3 \\
\hline $\mathrm{N}_{\text {irr }}$ & ** & $* *$ & $* *$ \\
\hline $\mathrm{N}_{\text {irl }}$ & $* *$ & $* *$ & *** \\
\hline $\mathrm{N}_{\text {sub }}$ & $3,83 \times 10^{10}$ & $3,01 \times 10^{10}$ & $3,17 \times 10^{10}$ \\
\hline $\mathrm{N}_{\mathrm{tr}}$ & $3,18 \times 10^{11}$ & $2,90 \times 10^{11}$ & $2,97 \times 10^{11}$ \\
\hline
\end{tabular}

*O valor negativo representa deformação de compressão.

** A equação utilizada fornece $\mathrm{N}$ para uma deformação de tração.

Nota-se que diferente do Perfil (3a), para o Perfil (3b), as duas primeiras camadas do pavimento sofreram compressão em sua fibra inferior, impossibilitando o cálculo do $\mathrm{N}_{\text {irr }}$ e do $\mathrm{N}_{\text {irl }}$.

Talvez pela inexistência de uma equação para a base, o Perfil (3b) apresentou valores de $\mathrm{N}$ mais de 1.000 vezes superiores aos valores encontrados para o Perfil (3a), ao 


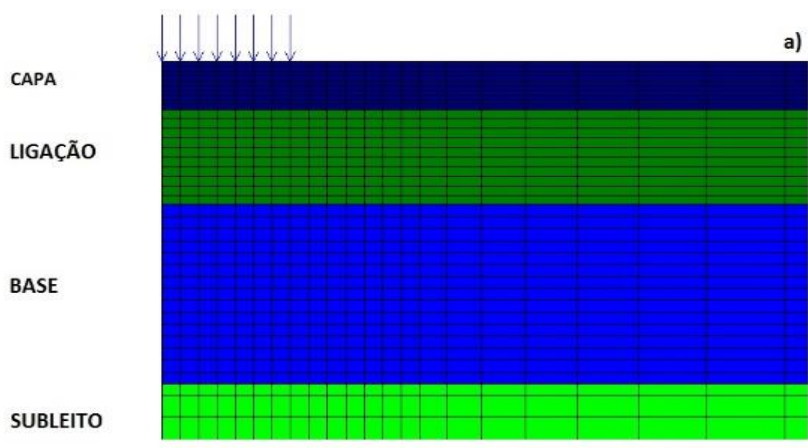

(a) Malha e camadas
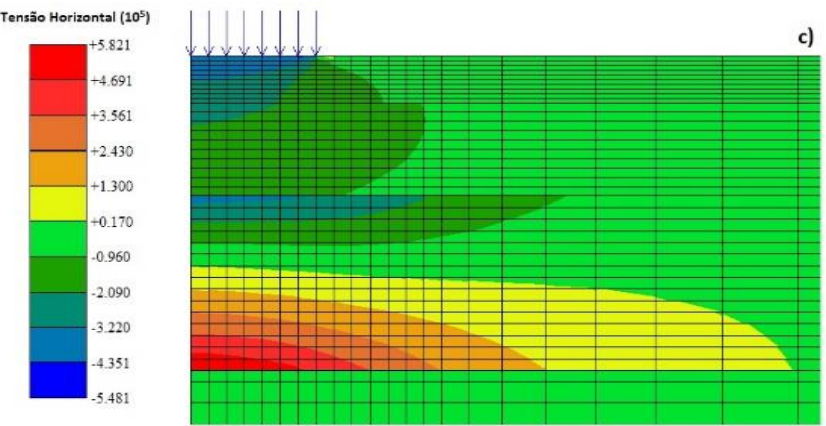

(c) Tensão horizontal $(\mathrm{Pa})$
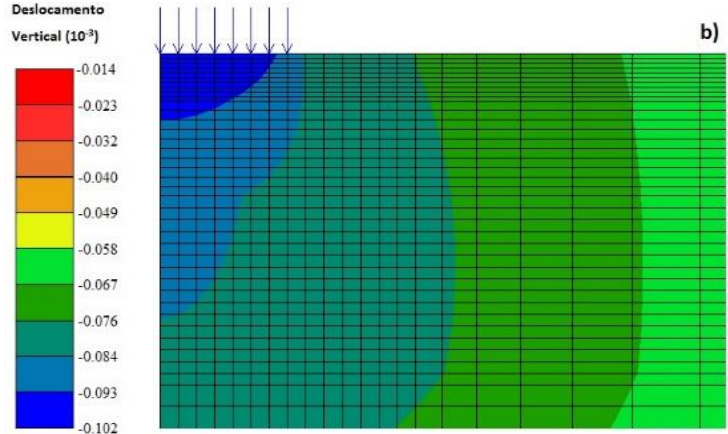

(b) Deslocamento vertical $(\mathrm{m})$
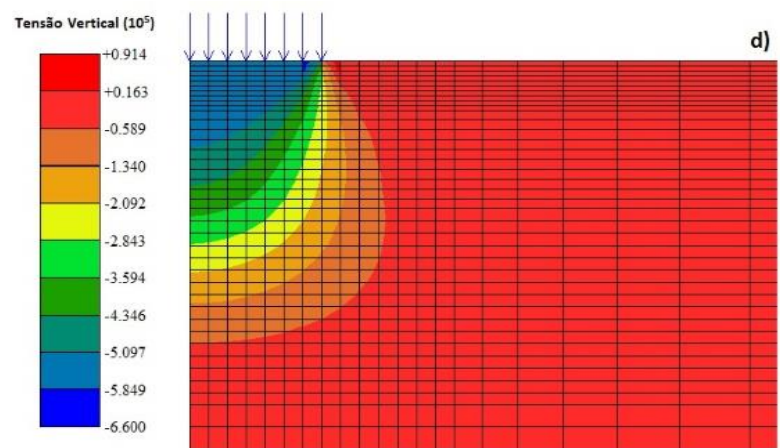

(d) Tensão vertical $(\mathrm{Pa})$

Figura 5. Detalhe da região próxima à carga para o Perfil (3b) que utiliza a Mistura I como camada de base

considerar o ponto de ruptura do pavimento, da ordem de $\mathrm{N}$ $=10^{10}$ para o topo do subleito $\left(\mathrm{N}_{\text {sub }}\right)$, sendo recomendado para rodovias com alto volume de tráfego. Na Figura 5, os resultados obtidos para o deslocamento vertical e para as tensões horizontal e vertical são apresentados para melhor análise da estrutura do Perfil (3b) cuja Mistura I compõe a camada de base.

Com relação ao Perfil (3b), assim como no Perfil (3a) é possível perceber que a concentração de tensão horizontal de tração está localizada na fibra inferior da base, a qual é constituída de MARQ com elevado percentual de fresado. Apesar disso, caso considerada no cálculo do dimensionamento pelo modelo da Equação 4, assim como realizada para as camadas de rolamento e de ligação (revestimento da estrutura), a deformação específica horizontal de tração da base seria igual a $2,50 \times 10^{-5}$, resultando em um $\mathrm{N}$ de $1,59 \times 10^{10}$. Ademais, a tensão vertical é dissipada, iniciando seu valor mínimo e constante na metade inferior da base.

Os perfis avaliados apresentam uma espessura do revestimento $20 \%$ menor do que o valor recomendado pelo DER-SP (2006), que é de $12 \mathrm{~cm}$ para um $\mathrm{N}$ de $1 \times 10^{7}$ a $2,5 \times 10^{7}$, intervalo encontrado para o Perfil (3a), e de $15 \mathrm{~cm}$ para um $\mathrm{N}$ maior que $5 \times 10^{7}$, resultado encontrado para o Perfil (3b) (DER-SP, 2006). A estrutura total do Perfil (3a), porém, foi $40 \%$ mais esbelta se comparada a estrutura obtida através do uso do dimensionamento empírico pelo método do DNIT por Benevides (2000) e por Benevides (2005), para a BR - 116 - Fortaleza - Pacajus, a qual apresenta $\mathrm{N}$ da mesma ordem de grandeza da estrutura aqui avaliada. Tal redução foi semelhante ao resultado encontrado para a estrutura avaliada por Freitas e Motta (2008).

O Perfil (3b) apresenta uma estrutura de mesma dimensão se comparada a estrutura apresentada por Magalhães (2004) quando a solução apresenta camada de ligação constituída por EME com RASF e $13 \%$ mais delgada quando a solução apresenta camada de ligação composta por EME com EVA. O tráfego que a estrutura do Perfil (3b) é capaz de suportar, porém, foi 100 vezes superior ao $\mathrm{N}$ encontrado pelas duas estruturas avaliadas por Magalhães (2004). Os resultados de $\mathrm{N}$ mostram que o Perfil (3b) pode apresentar uma melhor alternativa com o uso das MARQ com elevado percentual de RAP incorporado em camadas de base. A Mistura I, constituída quase que integralmente por RAP, pode proporcionar uma maior economia de materiais virgens se comparada as Misturas II e III.

\section{CONCLUSÕES}

Neste trabalho, foi realizada uma avaliação de diferentes MARQ com incorporação de elevado percentual de RAP (maior que 75\%) como alternativa para uso em camadas de elevado módulo de elasticidade em pavimentos flexíveis. Por meio de uma análise estrutural realizada pelo PMG/CAP3D, o qual utilizada o MEF para a geração da malha, foi determinado o tráfego que uma estrutura pré-estabelecida poderia suportar. A partir desse método foi possível calcular as deformações, os deslocamentos e as tensões em diferentes locais da estrutura, o que não seria possível realizar através do uso do atual método de dimensionamento brasileiro, que utiliza o CBR como parâmetro. Foram realizadas análises estruturais seguindo recomendações da literatura e do DER-SP.

Acredita-se que MARQ com a incorporação de alto percentual de reciclado poderiam ser uma alternativa para uso em camadas de bases e de ligação para pavimentos especiais, os quais necessitariam de EME. O uso desses materiais com essa aplicação é viável devido à consistência elevada do CAP oxidado oriundo dos resíduos, proporcionando às misturas asfálticas valores de MR superiores a $10.000 \mathrm{MPa}$. 
Misturas Asfálticas Recicladas a Quente com Incorporação de Elevado Percentual de Fresado Como Alternativa para Camada de Módulo Elevado

Essa alternativa pode apresentar vantagens como: pavimentos mais delgados se comparados a estruturas com camadas granulares (reduzindo em torno de $40 \%$ a espessura total do pavimento se comparado a um pavimento convencional com o mesmo nível de tráfego), além de reduzir o potencial de dano por fadiga e minimizar as deformações por tração no fundo da camada de revestimento. A execução de pavimentos mais delgados poderia proporcionar economia de recursos naturais (agregados e CAP), de transporte da usina à obra e de tempo de execução de camadas e redução na emissão de poluentes. Essa economia é dada principalmente pela reutilização de praticamente todo o material, principalmente o CAP, o qual geralmente é o insumo mais oneroso na produção de misturas asfálticas.

As alternativas que usam a Mistura I podem proporcionar uma maior economia de materiais virgens se comparada as Misturas II e III sem haver perda do ponto de vista estrutural. O Perfil (3b), o qual apresenta MARQ com a incorporação de alto percentual de fresado como camada de base, representou uma melhor solução considerando a análise estrutura realizada se comparado ao Perfil (3a), que utiliza essas misturas asfálticas como camada de ligação. Os Perfis (3a) e (3b) poderiam ser usados em rodovias de médio e alto volume de tráfego, respectivamente. Dessa maneira, acredita-se que a melhor alternativa analisada seria aquela representada pelo Perfil (3b) que utiliza a Mistura I. No entanto, sugere-se que o modelo seja refinado para esse tipo de pavimento, já que há pouca informação na literatura sobre o dimensionamento mecanístico-empírico de pavimentos especiais, e que seja realizada uma análise viscoelástica para os materiais asfálticos utilizados.

\section{AGRADECIMENTOS}

Os autores agradecem à empresa Insttale Engenharia pelo fornecimento do fresado e do refugo da usina, à Pedreira OCS pelo fornecimento dos agregados virgens, à Petrobras pelo fornecimento do CAP adotados na pesquisa e ao CNPq pelas bolsas de pesquisa fornecidas aos autores.

\section{REFERÊNCIAS}

Aragão, F. T. S.; Lee, J.; Kim, Y.; Karki, P. (2010) Materialspecific effects of hydrated lime on the properties and performance behavior of asphalt mixtures and asphaltic pavements. In: Construction and Building Materials, vol. 24, p. 538-544. DOI: 10.1016/j.conbuildmat.2009.10.005.

Balay, J. M.; Brosseaud, Y.; Bara, B.; Castaneda, E. (2012) Adaptation of the french pavement design to countries in South America. In: Congrès 8ème jornadas international des Asfalto, França.

Balbo, J. T.; Bodi, J. (2004) Reciclagem a quente de misturas asfálticas em usinas: alternativa para bases de elevado módulo de elasticidade. In: XVIII Congresso de Pesquisa e Ensino em Transportes (ANPET), Florianópolis - SC.

Benevides, S. A. S. (2000) Análise Comparativa dos Métodos de Dimensionamento de Pavimentos Asfálticos: Empírico do DNER e da Resiliência da COPPE/UFRJ em Rodovias do Estado do Ceará. Tese Mestrado, Programa de Pós-Graduação de Engenharia da Universidade Federal Do Rio De Janeiro, Rio de Janeiro - RJ.

Benevides, S. A. S. (2005) Análise comparativa dos métodos de dimensionamento de pavimentos asfálticos: empírico do DNER e da resiliência da COPPE/UFRJ em rodovias do estado do Ceará. Tese de Doutorado, Programa de Pós-Graduação de Engenharia da Universidade Federal Do Rio De Janeiro, Rio de Janeiro - RJ.

Bernucci, L. B.; Motta, L. M.; Cerati, J. A. P.; Soares, J. B. (2007) Pavimentação asfáltica: formação básica para engenheiros. $3^{\text {a }}$ Edição. Rio de Janeiro - RJ.

Botelho, R. P.; Cavalcante, R. N. A.; Coutinho, R. P.; Holanda, A. S., Parente JR, E. (2009) Desenvolvimento de um sistema gráfico para análise numérica de pavimentos asfálticos. In: IV Simpósio Internacional de Avaliação de Pavimentos e Projetos de Reforço, Fortaleza, CE, p. 1-12.

Brosseaud, Y. (2001) Reciclagem de misturas asfálticas: evolução após 20 anos e a situação atual da França. In: VII Congresso Brasileiro de Rodovias e Concessões (CBR\&C), Foz do Iguaçu - PR.

Cavalcante, R. N. A.; R. P. Coutinho; A. S. Holanda, Parente Jr, E. (2008) Gerador de malha bidimensional para análise de tensões e deformações em pavimentos asfálticos. In: XXIX Iberian Latin American Congress on Computational Methods in Engineering, CILAMCE, p. 1-18.

CEDEX - Centro de Estudios y Experimentación de Obras Públicas (2012). Reciclado en central en caliente. Disponível em: <http://www.cedexmateriales.es/catalogo-deresiduos/33/reciclado-de-pavimentos-asfalticos/valorizacion/164/demolicion-.html> acessado em 01/02/2016.

Corté, J. F.; Guidoux, Y. (1998) Service d'études techniques des routes et autoroutes e laboratorie central desponts et chaussées. Catalogue des Structures Types de Chaussées Neuves. Bagneux: Ministère de L'Equipement, des Transport et du Logement,

Corté, J. F. (2001) Development and uses of hard grade asphalt and high modulus asphalt mixes in France. Transportation Research Circular n. 503, p. 12-31.

Coutinho R. P.; Cavalcante, R. N. A.; Holanda, A. S., Parente Jr, E. (2008) Desenvolvimento de um gerador de malhas para análise de pavimentos pelo método dos elementos finitos. In: XXII Congresso de Pesquisa e Ensino em Transportes, ANPET, Fortaleza-CE, p. 1-12.

DER-SP (2006) IP-DE-P00/001 Projeto de pavimentação. Instrução de Projeto. São Paulo, SP.

DNER-PRO 011/79 (1979). Avaliação estrutural dos pavimentos flexíveis - Procedimento "B". Rio de Janeiro - RJ.

Engineers Journal (2014). Pavement mix is an 'overnight' success on Dublin's streets. Disponível em: <www.engineersjournal.ie/dublin-city-councilpavements/i> acessado em 04/02/2016.

Freitas, H. B. F; Motta, L. M. G. (2008) Uso de escória de aciaria em misturas asfálticas de módulo elevado. In: Revista Transportes. vol. 16, n. 2, p. 5-12.

DOI:10.14295/transportes.v16i2.22.

Holanda, A. S.; Parente Jr., E.; Araújo, T. D. P.; Melo, L. T. B.; Evangelista Jr, F., Soares, J. B. (2006) Finite elemento modeling of flexible pavements. In: XXVII Iberian Latin-American Congress on Computational Methods in Engineering (CILAMCE), Belém - PA. 
Keijzer, E. E., Leegwater, G. A., Vos-Effting, S. E., Wit, M. S. (2015) Carbon footprint comparison of innovative techniques in the construction and maintenance of road infrastructure in The Netherlands. In: Environmental Science \& Policy vol. 54 p. 218 225. DOI:10.1016/j.envsci.2015.06.010.

Ma, T.; Wang, H.; Huang, X.; Wang, Z.; Xiao, F. (2015) Laboratory performance characteristics of high modulus asphalt mixture with high-content RAP. In: Construction and Building Materials, vol. 101 p. $975-982$. DOI:

10.1016/j.conbuildmat.2015.10.160.

Magalhães, S. (2004) Misturas asfálticas de módulo elevado para pavimentos de alto desempenho. Tese de Doutorado, Universidade do Rio de Janeiro, Rio de Janeiro - RJ.

Mallick, R. B.; Kandhal, P. S.; Bradburry, R. L. (2008) Using warm mix asphalt technology to incorporate high percentage of reclaimed asphalt pavement (RAP) material in asphalt mixes. In: 87th TRB - Annual Meeting of the Transportation Research Board, Washington, Estados Unidos. DOI: 10.3141/2051-09.

Maupin, G. W.; Diefenderfer, B. K. (2006) Design of a highbinder-high-modulus asphalt mixture. Final Report Virginia Transportation Research Council VTRC 07-R15.

Motta, L. M. G.; Fritzen, M. A.; Vianna, A. A. D.; Ávila, G. (2011) Avaliação do comportamento de dois tipos de revestimentos asfálticos, dosados pelo método SUPERPAVE, sob ação de tráfego real na rodovia BR/040 - trecho concessionária Concer. Apresentação de Slides, In: VI Workshop Desenvolvimento Tecnológico nas Concessões Rodoviárias, Brasília - DF.

Muthadi, N. R., \& Kim, Y. R. (2008) Local calibration of mechanistic-empirical pavement design guide for flexible pavement design. In: Transportation Research Record: Journal of the Transportation Research Board, vol. 2087, p. 131-141. DOI: 10.3141/2087-14

NCHPR 1-37 (2004) National Cooperative Highway Research Program. Guide for mechanistic-empirical design of new and rehabilitated pavement structures". Transportation Research Board. Washington DC. National Research Council.

Newcomb, D.; Willis, R.; Timm, D. (2010) Perpetual asphalt pavements; a synthesis, In: Asphalt Pavement Alliance, Lanham, Maryland, Estados Unidos.

Njock, P. G. A. N.; Yueguang, L. (2015) Comparative study of french and chinese asphalt pavement design methods. In: Journal of Applied Science vol 15, p. 923-928. DOI:

10.3923/jas.2015.923.928

OC 24/2008 (2008) Pliego de Prescripciones Técnicas Generales para Obras de Carreteras y Puentes (PG-3). Artículos: 542- Mezclas bituminosas en caliente tipo hormigón bituminoso, Madrid, Espanha.

Oliveira, J. A. (2013) Avaliação do desempenho de misturas asfálticas recicladas mornas em laboratório e em campo". Dissertação de Mestrado, Petran / UFC - Universidade Federal do Ceará, Departamento de Engenharia de Transportes, Fortaleza $-\mathrm{CE}$.

Perret, J.; Ould-Henia, M.; Dumont, A. G. (2004) High modulus pavement design using Accelerated Loading Testing (ALT) In: 3rd Eurasphalt \& Eurobitume Congress, Viena, Áustria.
Pinto, S. e Preusller, E.S. (2010) Pavimentação Rodoviária: Conceitos Fundamentais sobre Pavimentos Flexíveis. $2^{\mathrm{a}}$ Edição. Rio de Janeiro: Synergia Editora, IBP.

Rohde, L. (2007) Estudo de misturas asfálticas de módulo elevado para camadas estruturais de pavimentos. Tese de Doutorado, Universidade Federal do Rio Grande do Sul, Porto Alegre - RS.

Selvi, P. (2015) Fatigue and rutting strain analysis on lime stabilized subgrades to develop a pavement design chart. In: Transportation Geotechnics, vol. 2 p. 86-98. DOI:10.1016/j.trgeo.2014.11.001.

Silva, S. A. T; Vidal, P. J. F; Holanda, A. S.; Parente Jr, E. (2013) Análise viscoelástica de pavimentos asfálticos utilizando elementos finitos e infinitos. In: Revista Transportes v.1, n. 3. DOI:10.4237/transportes.v21i3.676.

Timm, D.; Newcomb, D. (2016) Perpetual pavement design. An introduction to the PerRoad program In: Michigan Asphalt Conference, Michigan, Estados Unidos.

Villacorta, F. L. (2005) Diseño de una estructura de pavimento perpetuo (caso de estudio de una ruta nacional en Costa Rica). In: XIII Congresso Iberolatinoamericano del asfalto (CILA), San José, Costa Rica.

Zaumanis, M., Mallick, R. B., Frank, R. (2014) 100\% recycled hot mix asphalt: a review and analysis. In: Resources, Conservation and Recycling vol. 92, p. 230-245. DOI: 10.1016/j.resconrec.2014.07.007. 